\title{
Research on Characteristics of Chaotic Motion Based on the Wavelet Ridge
}

\author{
He Li ${ }^{1,2, ~ a}$, Jidan Wen ${ }^{1, b}$, Jie Zhang ${ }^{1, c}$ and Bangchun Wen ${ }^{1, d}$ \\ ${ }^{1}$ The State Key Laboratory of Mechanical System and Vibration, Shanghai 220240, China \\ ${ }^{2}$ School of Mechanical Engineering \& Automation, Northeastern University, Shenyang 110004 , \\ China \\ ahli@mail.neu.edu.cn, b wenjidan0601013403@163.com, ${ }^{\mathrm{c}}$ zj8651731@126.com, ${ }^{\mathrm{d}}$ \\ bcwen1930@vip.sina.com
}

Keywords: Wavelet ridges; wavelet transform; chaos; Crazy Climber algorithm

\begin{abstract}
The wavelet ridge method to analyze chaos is described, and the wavelet ridge method is applied to analysis of the nonlinear vibration of blooming mill which exists chaos. The results show that the wavelet ridge can tell the periodic motion, quasi-periodic motion or chaotic motion by analysising the time history of one component of the system state variables. Compared to the other researching methods, such as the Poincare sections or the phase diagram, we can find the wavelet ridge is more suitable to high dimensional chaotic systems and the clutter of instantaneous frequency which is represented by the wavelet ridge can distinguish between strong and week chaos motion. And it can provide more accurate partial details and features of chaotic motion.
\end{abstract}

\section{Introduction}

Chaotic state widely exists in natural phenomenon and social phenomenon. Chaotic science and other scientific interpenetrate each other, and there widely exists chaotic motion in various fields. In recent years, many scholars took research in the analysis method of chaotic motion. They proposed several methods, such as, Poincare section diagram, Lyapunov index method, phase diagram[1]. The modern methods are association index saturated method and the improved biggest Lyapunov index method[2]. They take the normalized variance logarithm value of predicting error as characteristics, and utilize the method of CAO to determine embedding dimension[3]. Wavelet is a kind of multiscale analysis method, and is widely used in the extraction of signal features[4-5].

Wavelet ridge line has one to one correspondence with the transient frequency of signals. If wavelet ridge line is known, the transient frequency of signals will also be known. This paper analysed chaotic motion by using the method of wavelet ridge, and was used in the analysis of blooming mill nonlinear vibration. Research shows that, the method of wavelet ridge only needs take research to an certain component time course of system status variables, and it can distinguish the periodic motion, quasi-periodic motion and chaotic motion of systems. If this method is compared with Poincare section diagram and phase diagram, it can be found that wavelet ridge line is more compatible with the research of high dimensional chaotic systems, and the chaotic degree of transient frequency which is represented by wavelet ridge line, can divide the chaotic motion into strong chaotic motion and weak chaotic motion, can provide the partial detail characteristics of chaotic motion more precisely, and can be propitious to the analysis, control and use of chaotic motion.

\section{Wavelet analysis and wavelet ridge theory}

Continuous wavelet transform and wavelet time-frequency localization characteristics. The expansion of function $f(t)$ under wavelet basis function in any spaces $L^{2}(R)$ is a continuous wavelet transform, and it can be expressed as, 


$$
\begin{aligned}
W T_{f}(a, b) & =\left\langle f(t), \psi_{a, b}(t)\right\rangle \\
& =\int_{-\infty}^{\infty} f(t) \bar{\psi}_{a, b}(t) \mathrm{d} t \\
& =\frac{1}{\sqrt{a}} \int_{-\infty}^{\infty} f(t) \bar{\psi}\left(\frac{t-b}{a}\right) \mathrm{d} t
\end{aligned}
$$

In Eq. 1, $\psi_{a, b}(t)$ represents the function system generated by expansion and translation of basic wavelet function; $\bar{\psi}_{a, b}(t)$ is the complex conjugate function of $\psi_{a, b}(t) ;\left\langle f(t), \psi_{a, b}(t)\right\rangle$ is the inner product of $f(t)$ and $\psi_{a, b}(t)$.

For wavelet function $\psi_{a, b}(t)$, its concentration area of time frequency window and translation on time axis will stretch along with the change of $a$ and $b$, but the area of concentration region won't change with $a$ and $b$. Generally speaking, $\psi_{a, b}(t)$ decreases rapidly, so the energy of wavelet function is concentrated in wavelet interval, and the product of time width and frequency width is very small. Wavelet is localized when it spreads in time and frequency domain.

Wavelet ridges and its extraction algorithm. Asymptotic signal under the meaning of Hilbert transform can be expressed as,

$$
f(t)=A_{f}(t) \cos \left(\varphi_{f}(t)\right)
$$

In Eq. 2, $A_{f}(t)=\left|z_{f}(t)\right| \quad, \quad z_{f}(t)$ are the analytic signal of $f(t)$; $\varphi_{f}(t)=\operatorname{Arg}\left(z_{f}(t)\right)=\arctan \left(f^{H}(t) / f(t)\right)$.

Instantaneous frequency is defined as,

$$
v(t)=\frac{1}{2 \pi} \frac{\mathrm{d} \varphi_{f}(t)}{\mathrm{d} t}
$$

The instantaneous frequency of signals reflects local frequency movement, and it is closely related to the phase information.

Progressive wavelet function $g(t)=A_{g}(t) \exp \left(i \varphi_{g}(t)\right)$ is selected to meet admissible condition. Assume that $t_{s}$ is the phase stagnation point of continuous wavelet transform integrand of signal $f(t)$. According to the concept of stagnation point, it can be obtained on the ridge line that,

$$
\left.\frac{\mathrm{d} \varphi_{a, b}(t)}{\mathrm{d} t}\right|_{t=t_{s}}=\varphi_{f}^{\prime}(b)-\frac{1}{a} \varphi_{g}^{\prime}(0)=0
$$

That is,

$$
a=a_{r}(b)=\frac{\varphi_{g}^{\prime}(0)}{\varphi_{f}^{\prime}(b)}
$$

In Eq. 5, $a_{r}(b)$ is wavelet ridge line, and it represents that wavelet ridge line is the function of translation parameter.

In this paper, the position corresponding to modulus maximum was found in the plane after continuous wavelet transform, and the method of penalty function was used in the condition of signal ridge, while Crazy Climber algorithm was used in extracting several ridges in signals. Molet wavelet function was used as wavelet generating function. 


\section{Wavelet ridge analysis with vibrating boundary self-excited system movement Dynamic model of blooming mill nonlinear vibration system.}

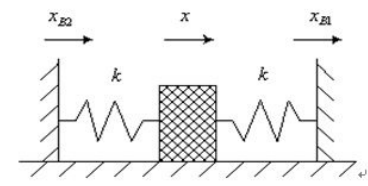

Fig. 1 Diagram of vibration system with a freedom degree

Blooming mill is simplified as an unidirectional nonlinear vibration system, and the fact that actual vibration system will be affected by many other conditions is considered. Assume that the equilibrium position when $x_{B 1}=x_{B 2}=0$ is origin. When $x \geq 0$, system vibration is just related to the right spring; when $x<0$, system vibration is just related to the left spring, and its disturbance form can be expressed as $x_{B 1}=\delta_{B 1} \sin \omega_{B 1} t$ and $x_{B 2}=\delta_{B 2} \sin \left(\omega_{B 2} t+\varphi\right)$. For blooming mill nonlinear vibration system, after dimensionless transformation, its differential equation can be expressed as

$$
\begin{cases}\ddot{y}-a \dot{y}+b \dot{y}^{3}+(y-\sin \bar{\omega} \tau)=0 & y \geq 0 \\ \ddot{y}-a \dot{y}+b \dot{y}^{3}+(y+\sin \bar{\omega} \tau)=0 & y<0\end{cases}
$$

The wavelet ridge analyses boundary perturbation frequency to the system response. Rolling mill vibration system shows rich nonlinear phenomenon when changes early by literature. The system appears periodic motion, quasi-periodic and chaotic motions with boundary perturbation frequency from 0.5 increases to 2.6. We use the method of wavelet ridge research sport under specific parameters of the system in order to get more and more accurate the information about system dynamics. Fig.2c shows the wavelet ridge of system speed. It includes four isolated wavelet ridge lines which are $\lambda=0.25, \lambda=0.50, \lambda=0.75$ and $\lambda=1.0$. These characteristics show that the system does cycle-4 movement under the parameters. Fig. $3 \mathrm{c}$ shows the wavelet ridge of system speed . It includes three isolated wavelet ridge lines which ridge lines in the position of each convention. These characteristics show that the system does quasi-periodic movement under the parameters. Fig. $4 \mathrm{c}$ shows system response in the given time has 1 2 more ridge line which hasn't sudden jumps phenomenon. The main ridge line is less, so the system movement under the parameters becomes weak chaos movement. Fig.5c shows system response in the given time has many ridge lines which have bifurcate phenomenon. The main ridge lines are more and messy, so system movement under the parameters becomes strong chaos movement.
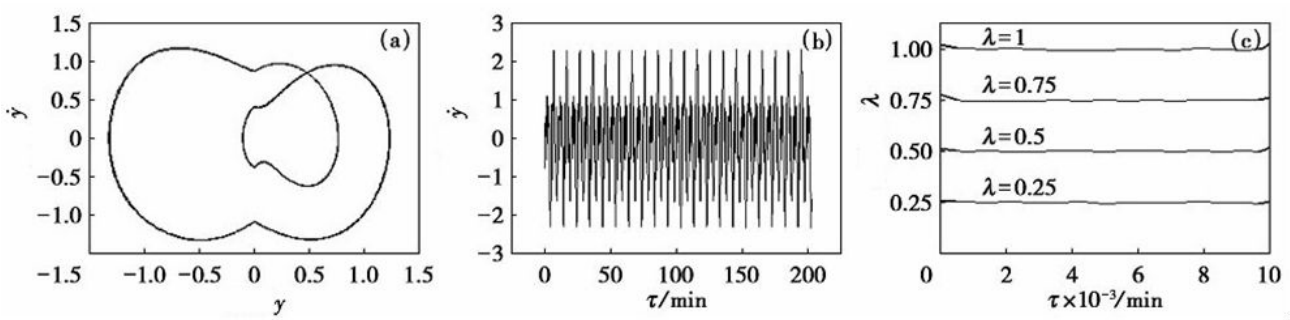

Fig. 2 Phase portrait, time history and diagram of instantaneous frequency of system when $\bar{w}=2$.

543812709
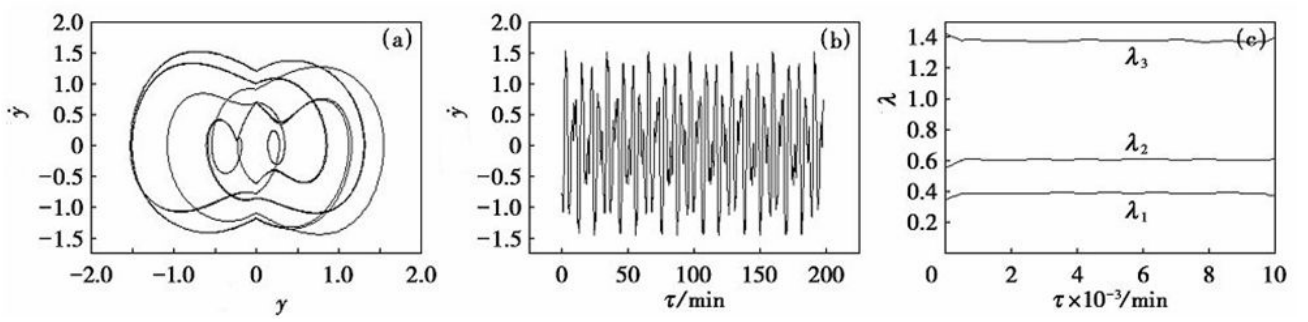

Fig. 3 Phase portrait, time history and diagram of instantaneous frequency of system when $\bar{w}=2.60$ 

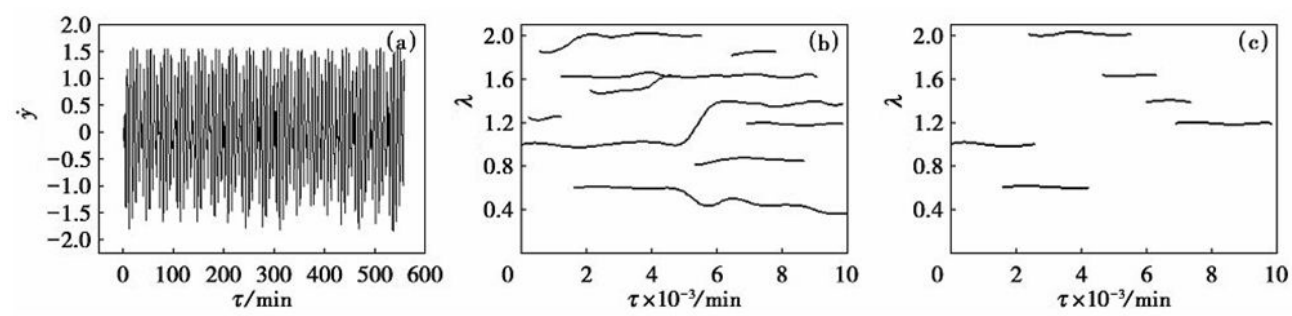

Fig. 4 Time history, diagram of instantaneous frequency and diagram of instantaneous main frequency of system when $\bar{w}=0.921405$
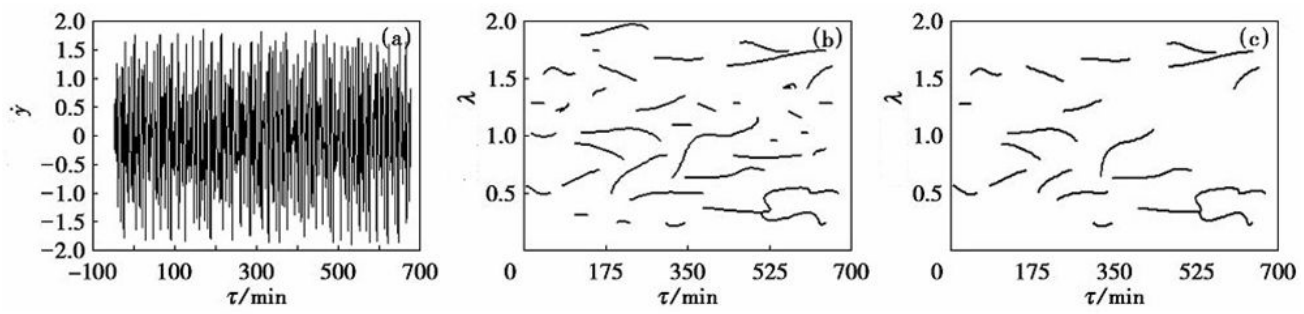

Fig. 5 Time history, diagram of instantaneous frequency and diagram of instantaneous main frequency of system when $\bar{w}=0.759866$

\section{Conclusion}

This paper puts forward the chaotic movement characteristics of the wavelet ridge line method. The wavelet ridge is applied to analysis of the nonlinear vibration of blooming mill which exists chaos. Discusses the rolling mill vibration system in early different boundary perturbation of frequency vibration characteristic compared to the other researching methods, we can find the wavelet ridge is more suitable to high dimensional chaotic systems and it can effectively distinguish the periodic motion to quasi-periodic, and chaotic motions by the wavelet ridge line and the clutter of instantaneous frequency which is represented by the wavelet ridge can distinguish between strong and week chaos motion. And it can provide more accurate partial details and features of chaotic motion. And it is more conducive to the chaotic motion analysis, control and utilization.

\section{Acknowledgements}

Supported by the New Century Excellent Talent Project of the Ministry of Education of China under Grant No NCET-10-0271, the Program for Changjiang Scholars and Innovative Research Team in University (PCSIRT), the Fundamental Research Funds for the Central Universities under Grant Nos N100503002 and N100703001, the Open Project of State Key Laboratory of Mechanical System and Vibration under Grant No MSV-2011-19.

\section{References}

[1] Yushu Chen, Andrew Y. T. Leung, Bifurcation and chaos in engineering. Springer-Verlag, Berlin, 1998.

[2] C. Chandre, S. Wiggins, T. Uzer, Time-frequency analysis of chaotic systems. Physica D, 181 (2003) 171-198.

[3] Luo GW, Xie JH, Bifurcation and chaos in a system with impacts, Physica D, 148(2001) 183-200.

[4] Argoul P., Le T-P., Instantaneous indicators of structural behaviour based on continuous Cauchy wavelet transform, Mechanical Systems and Signal Processing, 17(2003) 243-250.

[5] Chen zhang-wei, Pan hong, The Extraction Transient Frequency of Progressive Signal, Journal of Vibration Engineering, 14(1997) 203-209 